\title{
Direct oral anticoagulants in patients with atrial fibrillation following bariatric surgery: A single-center experience
}

\author{
Maksymilian Hanarz ${ }^{1}$, Aleksandra Gołąb ${ }^{2}$, Dariusz Plicner ${ }^{3,4}$, Anetta Undas ${ }^{5,6}$ \\ 'Faculty of Medicine, Jagiellonian University Medical College, Kraków, Poland \\ ${ }^{2}$ Faculty of Medicine and Dentistry, Pomeranian Medical University, Szczecin, Poland \\ ${ }^{3}$ Unit of Experimental Cardiology and Cardiac Surgery, Faculty of Medicine and Health Sciences, Andrzej Frycz Modrzewski Krakow University, Kraków, Poland \\ ${ }^{4}$ Department of Cardiovascular Surgery and Transplantation, John Paul II Hospital, Kraków, Poland \\ ${ }^{5}$ Krakow Center for Medical Research and Technologies, John Paul II Hospital, Kraków, Poland \\ ${ }^{6}$ Department of Experimental Cardiac Surgery, Anesthesiology and Cardiology, Institute of Cardiology, Jagiellonian University Medical College, Kraków, Poland
}

\author{
Correspondence to: \\ Dariusz Plicner, MD, PhD, \\ Unit of Experimental Cardiology \\ and Cardiac Surgery, \\ Faculty of Medicine \\ and Health Sciences, \\ Andrzej Frycz Modrzewski \\ Krakow University, \\ Herlinga-Grudzińskiego 1 \\ 30-705 Kraków, Poland, \\ phone: +48 122524503 , \\ e-mail: \\ plicner.dariusz@gmail.com \\ Copyright by the Author(s), 2021 \\ Kardiol Pol. 2021; \\ 79 (12): 1378-1381; \\ DOI: 10.33963/KP.a2021.0165 \\ Received: \\ October 9, 2021 \\ Revision accepted: \\ December 1, 2021 \\ Published online: \\ December 2, 2021
}

\section{INTRODUCTION}

Obesity is associated with an increased risk of atrial fibrillation (AF) [1], which largely requires lifelong anticoagulation to reduce stroke risk. Weight loss is recommended in the prevention of thromboembolism in AF patients with obesity [2].

Bariatric surgery has been proven to result in a significant weight loss, with associated reduction of obesity-related disability and mortality [3]. Sleeve gastrectomy (SG) and Roux-en-Y gastric bypass (RYGB) are the most common bariatric procedures [4], which alter the digestive tract function potentially affecting the pharmacokinetics of drugs, including anticoagulants $[2,5,6]$. Metabolism of vitamin K antagonists (VKA) seems to be less affected by bariatric surgery compared to direct oral anticoagulants (DOACs), and VKA may be a better choice in AF patients following such surgery $[5,6]$. A few studies on the effectiveness of DOACs in post-bariatric patients with AF yielded inconsistent results $[7,8]$.

We investigated whether DOACs are effective and safe in AF patients following bariatric procedures as compared to VKA and if plasma concentrations of DOACs could be useful in such patients.

\section{METHODS}

We studied 29 consecutive patients after laparoscopic SG or RYGB surgery, who developed $A F$ thereafter and were treated with VKA from 6 months to 5 years. They were referred to the Center for Coagulation Disorders in Kraków due to their preferences to receive DOAC instead of VKA in the years 2016-2019. The choice of a specific DOAC was based on patients' preferences (the once- vs. twice-daily regimen, access to the reversal agent, and the on-treatment risk of bleeding) and left at the physician's discretion. All patients on DOAC received standard-dose regimens and were tested for knowledge of AF and anticoagulation and subsequently enrolled in an educational program [9]. The control group included well-matched patients after the same bariatric procedures, who continued VKA therapy, started at the time of AF diagnosis. The exclusion criteria were cancer, renal failure, liver disease, severe thrombocytopenia, and dual antiplatelet therapy. Patients on aspirin were eligible. We assessed the thromboembolic risk using the $\mathrm{CHA}_{2} \mathrm{DS}_{2}$-VASC score [2]. Data on demographics, cardiovascular risk factors, comorbidities, and current treatment were collected. Comorbidities were defined in the Supplementary materials. The study was approved by the local Research Ethics Committee, which waived the need for informed consent.

After 4-8 weeks since the initiation of a DOAC, the plasma drug concentration was measured at 4-6 hours since the last dose as described in the Supplementary material. The time in the therapeutic range (TTR) was estimated as described [10].

During the follow-up, patients had ambulatory visits or were contacted by telephone every 6-12 months. We recorded ischemic stroke, transient ischemic attack, systemic embolism, major bleeding, clinically relevant 
Table 1. The characteristics of patients with atrial fibrillation after bariatric surgery

\begin{tabular}{|c|c|c|c|c|}
\hline \multirow[t]{2}{*}{ Variable } & \multirow{2}{*}{$\begin{array}{l}\text { All patients } \\
\quad(n=29)\end{array}$} & \multicolumn{3}{|c|}{ Patients receiving } \\
\hline & & $\begin{array}{c}\text { DOAC } \\
(n=14)\end{array}$ & $\begin{array}{c}\text { VKA } \\
(n=15)\end{array}$ & P-value \\
\hline Age, years & $56.9(4.3)$ & $57.0(3.6)$ & $56.7(5.0)$ & 0.87 \\
\hline Female, n (\%) & $7(24.1)$ & $4(28.6)$ & $3(20.0)$ & 0.68 \\
\hline Current smokers, n (\%) & $12(41.4)$ & $7(50.0)$ & $5(33.3)$ & 0.36 \\
\hline \multicolumn{5}{|l|}{ Bariatric procedure } \\
\hline Prior BMI, kg/m² & $45.2(4.0)$ & $45.3(3.4)$ & $45.1(4.6)$ & 0.86 \\
\hline Sleeve gastrectomy, $\mathrm{n}(\%)$ & $16(55.2)$ & $8(57.1)$ & $8(53.3)$ & 0.84 \\
\hline Roux-en-Y gastric bypass, n (\%) & $13(44.8)$ & $6(42.9)$ & $7(46.7)$ & 0.84 \\
\hline Weight reduction, \% & $19.3(4.8)$ & $19.2(4.9)$ & $19.4(4.9)$ & 0.92 \\
\hline \multicolumn{5}{|l|}{$\mathrm{AF}$} \\
\hline Time from surgery to AF diagnosis, years & $2.5(1.0-5.0)$ & $2.3(1.0-4.0)$ & $2.5(1.0-5.0)$ & 0.91 \\
\hline $\mathrm{CHA}_{2} \mathrm{DS}_{2}$-VASc score & $2.0(2.0-3.0)$ & $2.0(2.0-3.0)$ & $2.0(2.0-3.0)$ & 0.66 \\
\hline HAS-BLED score & $2.0(2.0-3.0)$ & $2.5(2.0-3.0)$ & $2.0(1.0-2.0)$ & 0.06 \\
\hline \multicolumn{5}{|l|}{ DOACs concentration after $4-6$ hours } \\
\hline Apixaban $(n=7), n g / m l$ & & $151.3(56.5)$ & & \\
\hline Rivaroxaban $(\mathrm{n}=4), \mathrm{ng} / \mathrm{ml}$ & & $129.8(49.6)$ & & \\
\hline Dabigatran $(\mathrm{n}=3), \mathrm{ng} / \mathrm{ml}$ & & $87.1(29.1)$ & & \\
\hline \multicolumn{5}{|l|}{ Expected peak DOAC concentration ${ }^{\mathrm{a}}$} \\
\hline Apixaban, ng/ml & & $69.0-321.0$ & & \\
\hline Rivaroxaban, ng/ml & & $178.0-343.0$ & & \\
\hline Dabigatran, ng/ml & & $52.0-383.0$ & & \\
\hline \multicolumn{5}{|l|}{ Follow-up } \\
\hline Duration, months & $29.4(5.4)$ & $29.9(6.5)$ & $29.0(4.3)$ & 0.68 \\
\hline Stroke, $\mathrm{n}(\%)$ & $2(6.9)$ & $1(7.1)$ & $1(6.7)$ & 0.96 \\
\hline $\mathrm{SE}, \mathrm{n}(\%)$ & $2(6.9)$ & $1(7.1)$ & $1(6.7)$ & 0.96 \\
\hline Major bleeding and CRNMB, n (\%) & $4(13.8)$ & $2(14.3)$ & $2(13.3)$ & 1.00 \\
\hline Death, $\mathrm{n}(\%)$ & $1(3.5)$ & 0 & $1(6.7)$ & 1.00 \\
\hline
\end{tabular}

Data are given as mean (standard deviation [SD]), median (interquartile range [IQR]), or number (percentage)

aBased on [5]

Abbreviations: AF, atrial fibrillation; ASA, acetylsalicylic acid; BMI, body mass index; CRNMB, clinically relevant non-major bleeding; DOAC, direct oral anticoagulants; $\mathrm{SE}$, systemic embolism; VKA, vitamin $\mathrm{K}$ antagonists

non-major bleeding, and mortality. The definitions of clinical outcomes were presented in the Supplementary materials.

Variables were presented as mean and standard deviations (SD), median (interquartile range [IQR]), or numbers (percentage) when appropriate. Normal distribution was tested using the Shapiro-Wilk test. Equality of variances was assessed using Levene's test. Differences between groups were compared using Student's, Welch's t-test, or Mann-Whitney $U$ tests based on the distribution of variables. Nominal variables were compared by Pearson's chi-square test or Fisher's exact test. A 2-tailed $P$-value $<0.05$ was considered statistically significant. Statistical analyses were performed with JMP ${ }^{\oplus}$, Version 15.2.0 (SAS Institute Inc., Cary, NC, USA).

\section{RESULTS AND DISCUSSION}

At the enrolment, the characteristics of AF patients, who underwent bariatric surgery ( $S G, n=16$, and RYGB, $n=13$ ) at the age of $53.7( \pm 4.5)$ years and either continued VKA therapy $(n=15)$ or were switched to DOAC $(n=14)$, were similar (Table 1). The most common comorbidities were dyslipidemia (86\%) and type 2 diabetes (73\%) (Supplementary material, Table S1). None of the patients had a prior stroke, diagnosed liver disease, or chronic kidney disease, stage 3 or higher. There was one major bleeding in a patient treated with acenocoumarol. After bariatric surgery, within the first year, the mean weight reduction was $19 \%$ (from a preoperative body mass index [BMI] of $45.2 \pm 4.0 \mathrm{~kg} / \mathrm{m}^{2}$, $\min$. 37.9, max. 51.4), with no difference related to the type of surgery. The median time from bariatric surgery to AF diagnosis was $2.5(1.0-5.0)$ years, and persistent AF was predominant ( $n=25,86 \%)$. Twenty-five (86\%) patients had $\mathrm{CHA}_{2} \mathrm{DS}_{2}$-VASc score $\geq 2$ points (min. 2, max. 5), while 4 patients had 1 point in this score.

Seven patients received apixaban $5 \mathrm{mg}$ twice a day, 4 rivaroxaban $20 \mathrm{mg}$ once a day, and 3 dabigatran $150 \mathrm{mg}$ twice a day. The peak DOAC plasma concentrations were, in most cases, within the reference range as suggested, but 2 patients receiving rivaroxaban (aged 54 and 59 years following SG and RYGB, respectively) had peak levels below the expected range $(74 \mathrm{ng} / \mathrm{ml}$ and $106 \mathrm{ng} / \mathrm{ml}$, respectively) [2]. Ten patients were treated with warfarin and five with acenocoumarol. Their mean TTR was $67( \pm 18) \%$ and 53\% 
of patients had TTR $>70 \%$. Both DOAC levels and TTR were unrelated to the type of surgery. Five patients in both groups declared the use of aspirin $75 \mathrm{mg}$ daily.

During a mean follow-up of $29.4( \pm 5.4)$ months, none of the patients were lost. The two patients receiving rivaroxaban, who had too low peak drug concentrations, were switched to apixaban or warfarin.

There were 4 thromboembolic events (5.8\% per year) including 2 ischemic strokes (Table 1), with no difference related to the type of anticoagulants or the surgery performed. A 56-year-old female following SG ( $\mathrm{CHA}_{2} \mathrm{DS}_{2}$-VASc 3) experienced a stroke after cessation of apixaban therapy while on prophylactic-dose enoxaparin due to severe pneumonia. Another 62-year-old female ( $\mathrm{CHA}_{2} \mathrm{DS}_{2}$-VASc 4) following SG was on acenocoumarol (TTR, 73\%) and experienced a stroke during viral infection with fever known to de-stabilize anticoagulation with VKA [12]. We also observed 2 systemic embolism events $\left(\mathrm{CHA}_{2} \mathrm{DS}_{2}\right.$-VASc 2 , each), both in men after RYGB, one on dabigatran and the other on warfarin (TTR, 42\%).

There were 2 major and 2 clinically relevant non-major bleeds (5.8\% per year); all in patients after RYGB and without previous severe bleeding. Two patients had major upper gastrointestinal bleeding, one on apixaban (plus aspirin) and one on acenocoumarol (TTR, 50\%). One patient treated with dabigatran (plus aspirin) and the other with acenocoumarol (TTR, 38\%) suffered from nonmajor bleeding.

Thromboembolic and bleeding events were recorded more frequently in patients following the RYGB procedure ( $6 / 8$ events, including 3 on DOACs and 3 on VKA; $P=0.04$ ). The TTR was lower in patients on VKA with those events (51\% vs. $73 \% ; P=0.03$ ). There were no relations in the occurrence of thromboembolic and bleeding complications among patients with TTR $>70 \%$ compared to the subjects with TTR $\leq 70 \%$.

One patient, free of thromboembolic or bleeding events, died of cancer after 37 months of the follow-up.

To our knowledge, this is the first Polish report on AF patients after RYGB or SG receiving DOAC, indicating that the risk of stroke/systemic embolism and bleeding is similar on DOAC versus VKA. Importantly, 6 out of 8 events were observed in patients following RYGB, therefore in our opinion, this subgroup should be closely monitored with the integrated approach by a multidisciplinary team [13]. As suggested by experts [5], the plasma DOAC concentration was measured, and the patients on apixaban and dabigatran had levels within the expected ranges with no differences related to the type of surgery. There are data suggesting caution while using rivaroxaban following RYGB [14], and, indeed, in 2 patients on rivaroxaban (one after RYGB), the peak drug concentration was below the expected value though the patients did not experience any complications and were switched to apixaban or VKA. The bioavailability of lipophilic rivaroxaban may be reduced following gastric bypass surgery when its absorption takes place distal to the stomach as discussed by Steffel et al. [5].

Hendricks et al. reported no difference in the occurrence of ischemic stroke/systemic embolism, major bleeding, and death between patients on DOAC and VKA in a bariatric cohort $(n=1673)$ [8]. In 29 bariatric patients on DOACs, investigators showed that bleeding events were common (29\%), with a significant rate of thromboembolic events (7\%); we recorded similar risks of those complications. In our study 2 outcomes were observed on apixaban and 2 on dabigatran. A relatively high risk of bleeding in bariatric patients is likely multifactorial, including over-the-counter aspirin use without a clear indication [11]. Two ischemic strokes were related to withdrawal of anticoagulation, which highlights the need for good compliance and education $[2,5,9]$.

Our study has several limitations. The size of the study group was small; however, AF is relatively uncommon among middle-aged bariatric patients. DOAC levels were not routinely measured during the follow-up.

This study suggests that DOAC could represent an acceptable alternative to VKA in AF patients after bariatric surgery when supported by plasma DOAC measurements at the initiation of anticoagulation.

\section{Supplementary material}

Supplementary material is available at https://journals. viamedica.pl/kardiologia_polska.

\section{Article information}

Conflict of interest: AU received lecture honoraria from Bayer, Boehringer Ingelheim, and Pfizer. Other authors declare no conflict of interest.

Open access: This article is available in open access under Creative Common Attribution-Non-Commercial-No Derivatives 4.0 International (CC BY-NC-ND 4.0) license, allowing to download articles and share them with others as long as they credit the authors and the publisher, but without permission to change them in any way or use them commercially. For commercial use, please contact the journal office at kardiologiapolska@ptkardio.pl.

How to cite: Hanarz M, Gołąb A, Pilcner D, Undas A. Direct oral anticoagulants in patients with atrial fibrillation following bariatric surgery: A single-center experience. Kardiol Pol. 2021; 79(12): 1378-1381, doi: 10.33963/KP.a2021.0165.

\section{REFERENCES}

1. Panchal G, Mahmood M, Lip GYH. Revisiting the risks of incident atrial fibrillation: a narrative review. Part 2. Kardiol Pol. 2019; 77(5): 515-524, doi: 10.33963/KP.14846, indexed in Pubmed: 31125027.

2. Hindricks G, Potpara T, Dagres N, et al. 2020 ESC Guidelines for the diagnosis and management of atrial fibrillation developed in collaboration with the European Association for Cardio-Thoracic Surgery (EACTS): The Task Force for the diagnosis and management of atrial fibrillation of the European Society of Cardiology (ESC) Developed with the special contribution of the European Heart Rhythm Association (EHRA) of the ESC. Eur Heart J. 2021; 42(5): 373-498, doi: 10.1093/eurheartj/ehaa612, indexed in Pubmed: 32860505.

3. Ministrini S, Mondovecchio G, Lupattelli G. New insights in pathophysiology of cardiovascular benefits after obesity surgery. Kardiol Pol. 2020; 78(7-8):657-658, doi: 10.33963/KP.15561, indexed in Pubmed: 32844611. 
4. Spaniolas K, Kasten KR, Brinkley J, et al. The changing bariatric surgery landscape in the USA. Obes Surg. 2015; 25(8): 1544-1546, doi: 10.1007/s11695-015-1764-x, indexed in Pubmed: 26072171.

5. Steffel J, Collins R, Antz M, et al. External reviewers. 2021 European Heart Rhythm Association Practical Guide on the Use of Non-Vitamin K Antagonist Oral Anticoagulants in Patients with Atrial Fibrillation. Europace. 2021; 23(10): 1612-1676, doi: 10.1093/europace/euab065, indexed in Pubmed: 33895845.

6. Martin KA, Lee CR, Farrell TM, et al. Oral anticoagulant use after bariatric surgery: a literature review and clinical guidance. Am J Med. 2017; 130(5): 517-524, doi: 10.1016/j.amjmed.2016.12.033, indexed in Pubmed: 28159600.

7. DeCamillo D, Haymart B, Barnes GD. Adverse events in patients taking apixaban or rivaroxaban who have undergone bariatric surgery: a retrospective case series. J Thromb Thrombolysis. 2021 [Epub ahead of print], doi: 10.1007/s11239-021-02573-6, indexed in Pubmed: 34559367.

8. Hendricks AK, Zieminski JJ, Yao X, et al. Safety and efficacy of oral anticoagulants for atrial fibrillation in patients after bariatric surgery. Am J Cardiol. 2020; 136: 76-80, doi: 10.1016/j.amjcard.2020.09.020, indexed in Pubmed: 32941819.
9. Janion-Sadowska A, Sadowski M, Konieczyńska M, et al. Polish regional differences in patient knowledge on atrial fibrillation and its management as well as in patterns of oral anticoagulant prescription. Kardiol Pol. 2019; 77(4):437-444, doi: 10.5603/KP.a2019.0036, indexed in Pubmed: 30835335.

10. Azar AJ, Cannegieter SC, Deckers JW, et al. A method to determine the optimal intensity of oral anticoagulant therapy. Thromb Haemost. 1993; 69(3): 236-239, indexed in Pubmed: 8470047.

11. Undas A, Drabik L, Potpara T, et al. Bleeding in anticoagulated patients with atrial fibrillation: practical considerations. Pol Arch Intern Med. 2020; 130(1):47-58, doi: 10.20452/pamw.15136, indexed in Pubmed: 31933483.

12. Self $T H$, Oliphant $C S$, Reaves $A B$, et al. Fever as a risk factor for increased response to vitamin $\mathrm{K}$ antagonists: a review of the evidence and potential mechanisms. Thromb Res. 2015; 135(1): 5-8, doi: 10.1016/j.thromres.2014.10.015, indexed in Pubmed: 25456000.

13. Pignatelli P, Violi F, Pastori D. Integrated care management of patients with atrial fibrillation-far from optimal. Pol Arch Intern Med. 2020; 130(3): 176-178, doi: 10.20452/pamw.15260, indexed in Pubmed: 32218392.

14. Rottenstreich A, Barkai $A, \operatorname{Arad} A$, et al. The effect of bariatric surgery on direct-acting oral anticoagulant drug levels. Thromb Res. 2018; 163: 190-195, doi: 10.1016/j.thromres.2017.11.006, indexed in Pubmed: 29157916. 